\title{
Characterization of Microstructural Inhomogeneities in Rolled Microalloyed Steels Based on EBSD Data.
}

\author{
Nikolay Y. Zolotorevsky ${ }^{1}$, Sergey N. Petrov ${ }^{2}$, Sergey N. Panpurin ${ }^{1}$, Alexander A. Kazakov ${ }^{3}$ and Olga \\ Pakhomova $^{3}$ \\ 1. Department of Mechanics and Control Processes, Polytechnical University, Saint-Petersburg, Russia. \\ 2. Central Research Institute of Structural Materials "Prometey", Saint-Petersburg, Russia. \\ 3. Metallurgical Technologies Department, Polytechnical University, Saint-Petersburg, Russia.
}

The method of quantitative metallography has been developed recently making it possible to evalutate bainitic microstructure of modern pipeline steels [1]. When using this method, the microstructural inhomogeneities identified as the blocks of bainite with lath-like morphology was revealed among different morphological forms elongated along the rolling direction. Moreover, these blocks were shown to reduce the essential mechanical properties of the plate. In the present study, the crystallographic features of such inhomogeneities was examined by means of the SEM using EBSD analysis in order to characterize them more rigorously and clarify their nature.

Industrially processed low-carbon microalloyed steel was investigated. Steel plate was hot rolled from a 300-mm slab to a 27.7-mm plate under industrial conditions. Last passes were conducted at nonrecrystallization temperatures $750 \pm 40^{\circ} \mathrm{C}$, and a true strain accumulated therewith was about 1.6. After rolling, the steel was cooled with a rate about $10 \mathrm{~K} / \mathrm{s}$. The samples for SEM examination were prepared from a central region of the steel plate. EBSD analysis was carried out by SEM Quanta 200 3D FEG using the EDAX Pegasus system with a step size $0.5 \mu \mathrm{m}$. The orientation relationship (OR) between $\alpha$ and $\gamma$ phases, the statistics of crystal misorientations and the orientations of the former austenite were determined for comprehensive characterization of the microstructure.

Fig. 1a presents an example of the microstructure, where the coarse-grained region corresponds to the inhomogeneity found previously by optical metallography. The differentiation of fine-grained and coarse-grained regions is supported in Fig. 1b by grain average misorientation (GAM) mapping. The scale of color differentiation used here was suggested recently to distinguish various $\alpha$-phase species [2]. One can see that the fine-grained regions are colored partially by blue, green and yellow. These colors indicate a relatively low level of crystal curvature. In particular, the blue color corresponds to the polygonal ferrite [2]. Alternatively, coarse-grained regions are colored in red (high level of crystal curvature). This corresponds to species formed by a displacive transformation and thus confirms that we are dealing with bainite.

Multiple variants of $\alpha$-phase lattice orientations may be produced within a single former austenite grain due to crystal symmetry. However, the so called "variant selection" leads to the predominance of certain inter-variant misorientations. Different kinds of bainite were shown to have different spectrums of the inter-variant boundaries [3-5]. The spectrum obtained in the specimen examined is shown in Fig. 2a (V1...V24 indicate possible inter-variant misorientations). It corresponds to the spectrums observed earlier for upper bainite [3-5]. This is supported by the ORs determined on the base of inter-variant misorientations for three regions (Fig. 2b). The ORs are shown here together with the ORs reported by Takayama et al. [3]. The latter ones were obtained for a bainite in low-carbon steel formed during isothermal holding (the holding temperatures are indicated at the plot). One can conclude from the 
comparison that we have the bainite formed at relatively high temperature.

Orientations of former austenite grains have been determined within the regions having microstructures of various types. They were found to correspond well to a rolling texture of FCC metals; most of them belong to the $\beta$-fiber. Therewith, the austenite, from which relatively fine-grained microstructure is produced, has orientations distributed uniformly within the $\beta$-fiber. Alternatively, the austenite, which produces the coarse-grained microstructural inhomogeneities, has orientations concentrated around the "brass component" $\{110\}<112>$. This observation can be explained by an orientation dependency of austenite deformation behavior, since peculiarities of the brass orientation - lower level of misorientations and greater homogeneity [6] - lead to a more strict variant selection during phase transformation, which as a result promotes formation of larger bainite packets as well as formation of bainite blocks with low misorientations.

\section{References:}

[1] A A Kazakov et al., CIS Iron and Steel Review, 2012, p. 4.

[2] A A Zisman, S N Petrov and A V Ptashnik, Metallurgist, 58 (2015), in press.

[3] N Takayama, G Miyamoto and T Furuhara, Acta Mater, 60 (2012), p. 2387.

[4] S N Panpurin et al., Materials Science Forum, 762 (2013), p. 110.

[5] N Y Zolotorevsky et al., Metal Sci. and Heat Treatment, 55 (2014), p. 550.

[6] L Delannay et al., Acta mater. 49 (2001), p. 2441
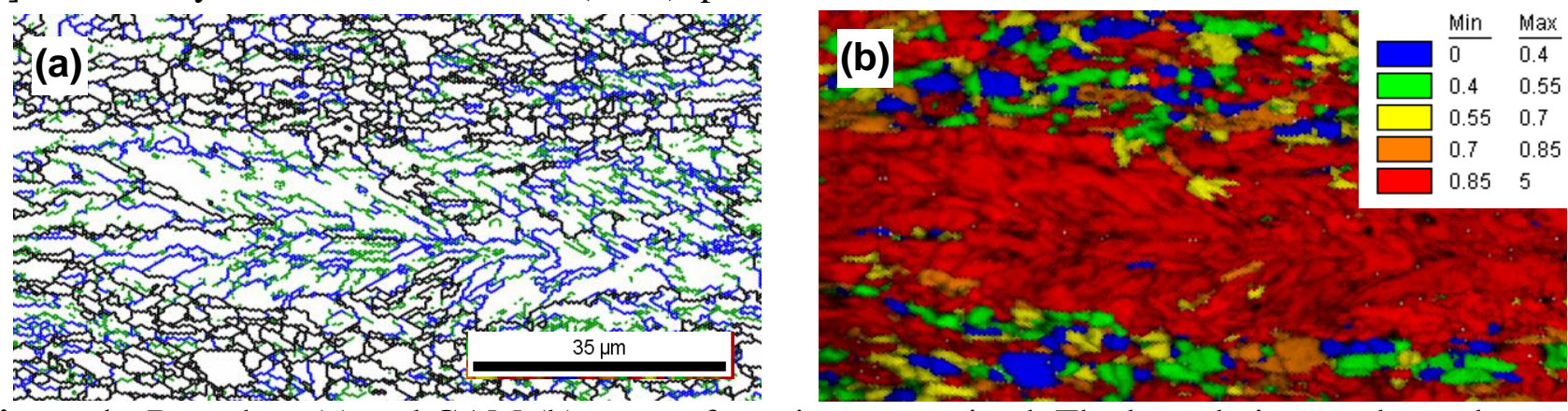

Figure 1. Boundary (a) and GAM (b) maps of specimen examined. The boundaries are drawn by green $\left(2^{\circ}<\theta<5^{\circ}\right)$, blue $\left(5^{\circ}<\theta<15^{\circ}\right)$ and black $\left(\theta>15^{\circ}\right)$ lines.

(a)

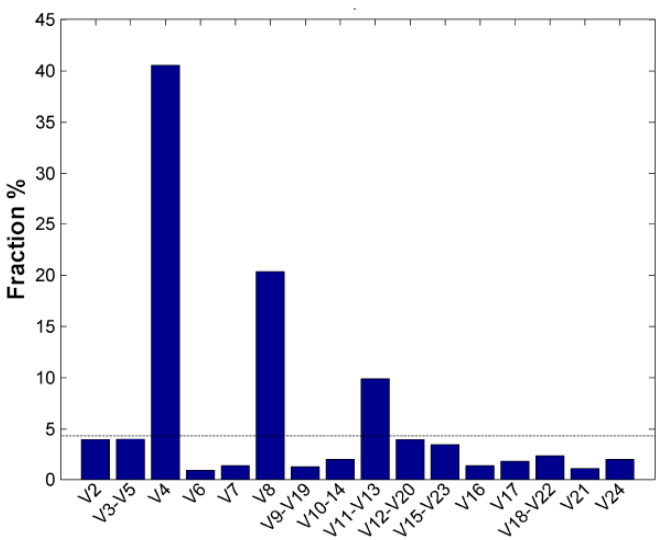

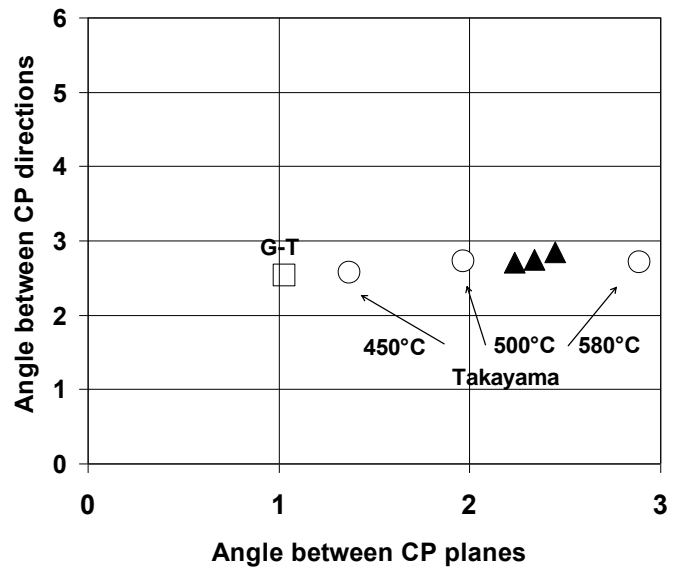

(b)

Figure 2. Length fractions of inter-variant boundaries (a) and the ORs presented by angles between planes $\{111\}_{\gamma}$ and $\{110\}_{\alpha}$ as a function of angles between directions $\langle 110\rangle_{\gamma}$ and $\langle 111\rangle_{\alpha}$ (black triangles) together with Greninger-Troiano (G-T) OR and the ORs reported by Takayama et all [3] (b). 